\title{
PROCEEDINGS
}

\section{Flattening, squeezing and the existence of random attractors}

\author{
By Peter E. Kloeden ${ }^{1}$ and José A. Langa ${ }^{2, *}$ \\ ${ }^{1}$ Institut fr Mathematik, Johann Wolfgang Goethe-Universitt, \\ 60054 Frankfurt am Main, Germany \\ ${ }^{2}$ Departmento de Ecuaciones Diferenciales y Análisis Numérico, Universidad de \\ Sevilla, Apartado de Correos 1160, 41080 Sevilla, Spain
}

The study of qualitative properties of random and stochastic differential equations is now one of the most active fields in the modern theory of dynamical systems. In the deterministic case, the properties of flattening and squeezing in infinite-dimensional autonomous dynamical systems require the existence of a bounded absorbing set and imply the existence of a global attractor. The flattening property involves the behaviour of individual trajectories while the squeezing property involves the difference of trajectories. It is shown here that the flattening property is implied by the squeezing property and is in fact weaker, since the attractor in a system with the flattening property can be infinite-dimensional, whereas it is always finite-dimensional in a system with the squeezing property. The flattening property is then generalized to random dynamical systems, for which it is called the pullback flattening property. It is shown to be weaker than the random squeezing property, but equivalent to pullback asymptotic compactness and pullback limit-set compactness, and thus implies the existence of a random attractor. The results are also valid for deterministic non-autonomous dynamical systems formulated as skew-product flows.

Keywords: flattening; random dynamical systems; random attractors; squeezing property

\section{Introduction}

The theory of dynamical systems has been successfully used from decades ago to analyse qualitative properties of many models of differential equations arising from Physics, Mechanics, Chemistry, Biology, etc. (See Hale 1988; Temam 1988; Ladyzhenskaya 1991; Vishik 1992; Chepyzhov \& Vishik 2002.) More recently, some of these ideas have also been used to describe the asymptotic behaviour of random and stochastic differential equations (Schmalfuss 1992; Crauel \& Flandoli 1994; Arnold 1998). In all cases, the concept of global attractor plays a crucial role. When the model is related to a system of partial differential equations, the existence of a global attractor is usually related to a kind of squeezing or flattening of the high modes in the evolution in time of trajectories, which leads to some of the most impressive results in the theory of infinite-dimensional dynamical * Author for correspondence (langa@us.es). 
systems. In this work, we want to go further in the clarification of the meaning of these kind of flattening properties and its relation with the existence of attractors, both in the deterministic and the stochastic cases.

It is often not difficult to show that a dynamical system given in terms of a specific differential equation has a bounded absorbing set. In finite-dimensions, such sets are compact and this is sufficient to ensure the existence of a global attractor. In the infinite-dimensional case, however, requiring an absorbing set to be compact is a severe restriction. An additional property to the existence of a bounded absorbing set is needed to ensure that there is a global attractor, such as the compactness, eventual compactness or asymptotic compactness of the flow operator (Hale 1988; Temam 1988; Ladyzhenskaya 1991; Vishik 1992; Rosa 1998; Robinson 2001).

An alternative idea is the squeezing property, which was introduced by Foias \& Temam in the context of the Navier-Stokes equations (Foias \& Temam 1979; Foias et al. 1988) and is applicable to many other classes of dissipative partial differential equations (Eden et al. 1994; Temam 1988; Robinson 2001). In the squeezing property, a finite-dimensional subspace of what are called lower order modes is introduced and either the higher order modes are bounded by the lower modes or the solutions are squeezed together. The squeezing property, which also requires the existence of a bounded absorbing set, has been used with considerable success to establish interesting properties of dissipative dynamical systems, such as determining modes (Foias \& Prodi 1967; Robinson 2001), the finite-dimensionality of global attractors (Constantin et al. 1985) and even to construct exponential attractors (Eden et al. 1994).

A related idea, which we shall call flattening, was introduced by Ma et al. (2002) under the name Condition (G). It assumes the existence of a bounded absorbing set and requires the finite-dimensional modes to become uniformly bounded with the remaining higher order modes becoming sufficiently small. Ma et al. (2002) showed that it is equivalent to a form of asymptotic compactness in uniformly convex Banach spaces, and that it implies the existence of a compact attractor. In most cases, it is not difficult to verify because estimates for the flattening property are obtained in much the same way as those needed to show that there is a bounded absorbing set. However, a major difference from the squeezing property is that the resulting attractor need not be finite dimensional.

In the first part of this paper, we will show that the flattening property is implied by the squeezing property in uniformly convex Banach spaces and then give counter examples that satisfy the flattening property but not the squeezing property - thus flattening is a weaker property than squeezing.

In the second and main part of the paper, we will extend the idea of flattening to random dynamical systems and compare it with pullback squeezing, the corresponding generalization of the squeezing property to deterministic nonautonomous dynamical systems. Our results make no use of the topology of the autonomous driving system of the skew product flow, therefore, apply equally well to deterministic non-autonomous dynamical systems formulated as skew product flows, e.g. systems generated by reaction diffusion equations with temporally almost periodic coefficients. In particular, we do not need to assume the existence of a uniform absorbing set as do Wang et al. (in press), who extend the flattening concept to deterministic skew product flows, thus, our results also generalize theirs in this context. 
We will use the following measure of non-compactness.

Definition 1.1. Let $X$ be a metric space and $D$ a bounded subset of $X$. The Kuratowski measure of non-compactness $\gamma(D)$ of $D$ is defined by $\gamma(D)=\{\inf \delta>0: D$ admits a finite cover by sets of diameter $\leq \delta\}$.

The following summarizes some of the basic properties of this measure of non-compactness (e.g. Deimling 1985).

Lemma 1.2. Let $X$ be a Banach space and let $\gamma$ be the measure of noncompactness. Then

(i) $\gamma(D)=0$ if, and only if, $\bar{D}$ is compact.

(ii) $\gamma\left(D_{1}+D_{2}\right) \leq \gamma\left(D_{1}\right)+\gamma\left(D_{2}\right)$.

(iii) $\gamma\left(D_{1}\right) \leq \gamma\left(D_{2}\right)$ for $D_{1} \subset D_{2}$.

(iv) $\gamma\left(D_{1} \bigcup D_{2}\right) \leq \max \gamma\left(D_{1}\right), \gamma\left(D_{2}\right)$.

(v) $\gamma(\bar{D})=\gamma(D)$.

(vi) If $F_{1} \supset F_{2} \ldots$ are non-empty closed sets in $X$ such that $\gamma\left(F_{n}\right) \rightarrow 0$ as $n \rightarrow \infty$, then $\bigcap_{n \geq 1} F_{n}$ is non-empty and compact.

In addition, let $X$ be an infinite-dimensional Banach space with a decomposition $X=X_{1} \oplus X_{2}$ and let $P: X \rightarrow X_{1}, Q: X \rightarrow X_{2}$ be projectors with $\operatorname{dim} X_{1}<\infty$. Then

(vii) $\gamma(B(\epsilon))=2 \epsilon$, where $B(\epsilon)$ is a ball of radius $\epsilon$.

(viii) $\gamma(D)<\epsilon$ for any bounded subset $D$ of $X$ for which the diameter of $Q D$ is less than $\epsilon$.

\section{The deterministic autonomous case}

The observed squeezing of high modes of the difference of trajectories in turbulent fluids has been formulated mathematically as the squeezing property by Foias \& Temam (1979), and has been used to prove many interesting and beautiful results for the Navier-Stokes equations and similar types of dissipative dynamical systems.

Definition 2.1 Squeezing property. Suppose that a semiflow $S$ on a Banach space $X$ has a bounded absorbing set $B$ in $X$. Let $P$ be a projection onto a finitedimensional subspace of $X$ and $Q=I-P$. Then for $x, y \in B$ either

$$
\|Q(S(1) x-S(1) y)\| \leq\|P(S(1) x-S(1) y)\|,
$$

i.e. the higher modes are bounded by the lower modes, or

$$
\|S(1) x-S(1) y\| \leq \delta\|x-y\|,
$$

for some $\delta \in(0,1)$, i.e. the solutions are squeezed together.

In applications to the planar Navier-Stokes equations, for example, $P$ is usually taken as the projector onto the subspace of $X$ spanned by the first $N$ eigenfunctions associated with the Stokes operator $A$, i.e. $P u=\sum_{i=1}^{N}\left(u, w_{i}\right) w_{i}$, where $\left\{w_{i}\right\}_{i=1}^{\infty}$ is the orthonormal basis in $X$ consisting of the eigenfunctions of $A$, and the operator $Q$ is defined as $Q u=\sum_{i=N+1}^{\infty}\left(u, w_{i}\right) w_{i}$. 
There is another, simpler feature of the dynamics in many dissipative systems in infinite-dimensional spaces, namely, the dynamics in the lower order modes becomes bounded and that in the higher modes becomes small. Ma et al. (2002) formulated this as Condition $(\mathrm{G})$ and showed that it is sufficient for the existence of a global attractor. We will call it the flattening property here.

Definition 2.2 Flattening property. Suppose that a semi flow $S$ on a Banach space $X$ has a bounded absorbing set $B$ in $X$. For any bounded set $D \subset X$ and for any $\epsilon>0$, there exists $T_{\epsilon}(D)>0$ a finite-dimensional subspace $X_{\epsilon}$ of $X$, and a bounded projector $P_{\epsilon}: X \rightarrow X_{\epsilon}$ such that $\bigcup_{t \geq T_{\epsilon}(D)} P_{\epsilon} S(t) D$ is bounded and

$$
\left\|\left(I-P_{\epsilon}\right) S(t) x_{0}\right\|<\epsilon, \quad \forall t \geq T_{\epsilon}(D), \quad x_{0} \in D .
$$

The flattening and squeezing properties obviously seem to be closely related and we want to clarify this relationship. We will show that the flattening property is a weaker concept than the squeezing property.

In $\S 2 a, b$, we will prove that squeezing implies flattening, at least when the Banach space $X$ is uniformly convex, i.e. for all $\epsilon>0$ there exists $\delta>0$ such that, given $x, y \in X,\|x\|,\|y\| \leq 1,\|x-y\|>\epsilon$, then $(\|x+y\| / 2)<1-\delta$. Requiring a space to be uniformly convex is not a severe restriction in applications, since this property is satisfied by all Hilbert spaces, the $L^{p}$ spaces with $1<p<\infty$, and most Sobolev spaces $W^{k, p}$ with $1<p<\infty$ (see Brézis 1983; section III.7). We also give counter examples that satisfy the flattening property but not the squeezing property.

\section{(a) Squeezing implies flattening}

Ma et al. (2002) introduced the following concept under the name of $\omega$-limit compact, but we will call it limit-set compact to avoid confusion in the stochastic setting later, where $\omega$ is used in another context.

Definition 2.3. A semi flow $S$ on a Banach space is said to be limit-set compact if for every bounded set $D \subset X$ and $\epsilon>0$ there exists a $T_{\epsilon}(D)>0$ such that

$$
\gamma\left(\bigcup_{t \geq T_{\epsilon}(D)} S(t) D\right)<\epsilon,
$$

where $\gamma$ is a measure of non-compactness defined on the subsets of $X$.

They then prove (theorem 3.10 in Ma et al. (2002)) that a semi-dynamical system is flattening if it is limit-set compact, provided $X$ is a uniformly convex Banach space.

The following result proves that the squeezing property is a sufficient condition for limit-set compactness. Thus, for uniformly convex Banach spaces $X$, flattening is indeed a weaker concept than squeezing.

Lemma 2.4. Suppose that $B=B_{X}(0, r)$, the ball in a Banach space $X$ of radius $r<0$ centred on the origin, is an absorbing set of a semi flow $S$ on $X$. If $S$ satisfies the squeezing property on $B$, then it is limit-set compact and thus has a global attractor in that B. Moreover, if $X$ is a uniformly convex Banach space, then $S$ has the flattening property. 
Proof. Without lost of generality, we can suppose $B$ is a positively invariant absorbing set, as, if not, we could consider $\bigcup_{t \geq t_{B}} S(t) B$ as a new bounded absorbing set for some $T_{B}$, such that $S(t) B \subset B$ for all $t \geq T_{B}$.

Using the squeezing property and lemma 2.1 in Eden et al. (1994), the set $S(1) B$ can be covered by $k_{0}$ balls of radius $r / 2$, in other words

$$
S(1) B \subset \bigcup_{i=1}^{k_{0}} B\left(a_{i}^{1}, r / 2\right) \text {. }
$$

In turn, each of the balls $B\left(a_{i}^{1}, r / 2\right)$ can be covered by $k_{0}$ balls of radius $r / 2^{2}$, so that

$$
S(2) B \subset \bigcup_{i=1}^{k_{0}^{2}} B\left(a_{i}^{2}, r / 2^{2}\right) \text {. }
$$

Iteratively, we get that

$$
S(n) B \subset \bigcup_{i=1}^{k_{0}^{n}} B\left(a_{i}^{n}, r / 2^{n}\right) .
$$

Now, given $\epsilon>0$, there exists $n_{0}$ such that $r / 2^{n}<\epsilon$ for all $n \geq n_{0}$; thus, $S\left(n_{0}\right) B$ can be covered by a finite number of balls of radius less than $\epsilon$. But note that

$$
S(t) B \subset S\left(n_{0}\right) B \text { for all } t \geq n_{0},
$$

so

$$
\gamma\left(\bigcup_{t \geq n_{0}} S(t) B\right)<\epsilon
$$

Finally, if $X$ is a uniformly convex Banach space, theorem 3.10 in Ma et al. (2002) implies the system is flattening.

\section{(b) Flattening does not imply squeezing}

A clear difference between flattening and squeezing is that the finitedimensional subspace $X_{\epsilon}$ of $X$ in the flattening property may depend on the choice of $\epsilon>0$, while the finite-dimensional projector $P$ and subspace $P X$ in the squeezing property are fixed at the start.

Systems with an infinite-dimensional global attractor are counter examples in which flattening holds but squeezing is impossible. By theorems 3.9 and 3.10 in Ma et al. (2002), the existence of a global attractor implies that the system is flattening, whereas the squeezing property is a sufficient condition for the finitedimensionality of a global attractor (Eden et al. 1994; Robinson 2001). Thus, the example in Chepyzhov \& Vishik (2002; pp. 161) is flattening, but not squeezing.

Actually, simpler examples involving infinite-dimensional systems of uncoupled ordinary differential equations (ODE) show this relationship much more directly. The infinite-dimensional space $\ell_{2}$ with the norm

$$
\|\boldsymbol{x}\|_{2}=\sqrt{\sum_{i=1}^{\infty}\left|x_{i}\right|^{2}}, \quad \boldsymbol{x}=\left(x_{1}, x_{2}, \ldots\right) \in \mathbb{R}^{\infty},
$$

is a Hilbert space (hence a uniformly convex Banach space). The infinitedimensional ODE

$$
\frac{\mathrm{d} x_{1}}{\mathrm{~d} t}=x_{1}\left(1-x_{1}^{2}\right), \quad \frac{\mathrm{d} x_{i}}{\mathrm{~d} t}=-x_{i}, \quad i=2,3, \ldots,
$$


in $\ell_{2}$ satisfies the squeezing property and has the global attractor (12)

$$
\boldsymbol{A}=[-1,1] \times \prod_{i \geq 2}\{0\},
$$

which is a one-dimensional compact subset of $\ell_{2}$. One the other hand, the infinitedimensional ODE (13)

$$
\frac{\mathrm{d} x_{i}}{\mathrm{~d} t}=x_{i}\left(i^{-2}-x_{i}^{2}\right), \quad i=1,2, \ldots,
$$

in $\ell_{2}$ satisfies the flattening property but not the squeezing property. It has the global attractor

$$
\boldsymbol{A}=\prod_{i \geq 1}\left[-i^{-1}, i^{-1}\right]
$$

which is an infinite-dimensional compact subset of $\ell_{2}$.

Remark 2.5. Chueshov \& Laisecka (2004) (see also Khanmamedov (2006)) write a sufficient condition, related to a kind of squeezing in the difference of two trajectories for the asymptotic compactness of a deterministic dynamical system. As we will show later, there exists an equivalence between the flattening and the asymptotically compact property of a system, so that the condition in Chueshov \& Laisecka (2004) would be also sufficient for the flattening property. It would be interesting to study if the contrary is also true or not.

\section{Random dynamical systems}

Let $(\Omega, \mathcal{F}, \mathbb{P})$ be a probability space and let $X$ be a Banach space. Arnold (1998) defined a random dynamical system $(\operatorname{RDS})(\theta, \phi)$ on $\Omega \times X$ in terms of a metric dynamical system $\theta$ on $\Omega$, which represents the noise driving the system, and a co-cycle mapping $\phi: \mathbb{R}_{+} \times \Omega \times X \rightarrow X$, which represents the dynamics in the state space $X$ and satisfies the properties

(i) $\phi\left(0, \omega, x_{0}\right)=\phi_{0}$ for all $x_{0} \in X$ and $\omega \in \Omega$,

(ii) $\phi\left(s+t, \omega, x_{0}\right)=\phi\left(s, \theta_{t} \omega\right) \phi\left(t, \omega, x_{0}\right)$ for all $s, t \geq 0, x_{0} \in X$ and $\omega \in \Omega$,

(iii) $\left(t, x_{0}\right) \mapsto\left(t, \omega, x_{0}\right)$ is continuous for each $\omega \in \Omega$, and

(iv) $\omega \mapsto \phi\left(t, \omega, x_{0}\right)$ is $\mathcal{F}$-measurable for all $\left(t, x_{0}\right) \in \mathbb{R}_{+} \times X$.

A metric dynamical system $\theta \equiv\left(\Omega, \mathcal{F}, \mathbb{P}, \theta_{t}, t \in \mathbb{R}\right)$ is a family of measure preserving transformations $\theta_{t}: \Omega \rightarrow \Omega, t \in \mathbb{R}$ such that $\theta_{0}=i d_{\Omega}, \theta_{t}^{\circ} \theta_{s}=\theta_{t+s}$ for all $t, s \in \mathbb{R}$, the map $(t, \omega) \mapsto \theta_{t} \omega$ is measurable and $\theta_{t} \mathbb{P}=\mathbb{P}$ for all $t \in \mathbb{R}$.

Random dynamical systems are generated by finite-dimensional differential equations with random coefficients or stochastic differential equations with a unique and global solution as well as by some infinite-dimensional stochastic evolution equations.

A family $\mathcal{D}=\left\{D_{\omega}, \omega \in \Omega\right\}$ of non-empty closed subsets of a Banach space $X$ is called a random closed set if the map $\omega \mapsto \operatorname{dist}\left(x, D_{\omega}\right)$ for each $x \in X$ is measurable with respect to $F$. Such a family $\mathcal{D}$ is said to be tempered if $D(\omega) \subset B_{X}(0, r(\omega))$ $\mathbb{P}$-a.s., where $r(\omega)$ is a tempered random variable, i.e. satisfying

for all $\epsilon>0$.

$$
\lim _{|t| \rightarrow+\infty} \frac{r\left(\theta_{t} \omega\right)}{\mathrm{e}^{\epsilon|t|}}=0, \mathbb{P} \text {-a.s. }
$$


The following concept of a random attractor for random dynamical systems (Schmalfuss 1992; Crauel \& Flandoli 1994; Arnold 1998; Crauel et al. 1995; Flandoli \& Schmalfuss 1996) extends that of a global attractor in autonomous deterministic systems to random dynamical systems.

Definition 3.1. A random compact set $\mathcal{A}=\left\{A_{\omega}, \omega \in \Omega\right\}$ of a Banach space $X$ is said to be a random attractor for an $\operatorname{RDS}(\theta, \phi)$ in $X$ if it is $\phi$-invariant, in other words

$$
\phi\left(t, \omega, A_{\omega}\right)=A_{\theta_{t} \omega}, \quad t \geq 0, \quad \mathbb{P} \text {-a.s. },
$$

where $\phi\left(t, \omega, A_{\omega}\right)=\bigcup_{a \in A_{\omega}} \phi(t, \omega, a)$, and pullback attracts every tempered random set $\hat{D}=\left\{D_{\omega}, \omega \in \Omega\right\}$ in $X$ in the sense that

$$
\lim _{t \rightarrow \infty} \operatorname{dist}_{X}\left(\phi\left(t, \theta_{-t} \omega, D_{\theta_{-t}}\right), A_{\omega}\right)=0,
$$

where $\operatorname{dist}_{X}(\cdot, \cdot)$ denotes the Hausdorff semidistance between subsets of $X$.

The following result (see Flandoli \& Schmalfuss 1996) ensures the existence of a random attractor for an RDS on a Banach space. A partial aim of the present paper is to establish the existence of a random attractor under weaker assumptions on the co-cycle mapping.

Theorem 3.2. Let $(\theta, \phi)$ be an $R D S$ on a Banach space $X$ such that $\phi(t, \omega, \cdot)$ : $X \rightarrow X$ is a compact operator for each fixed $t>0$ and $\omega \in \Omega$.

If there exists a tempered random set $\hat{\mathcal{B}}=\left\{B_{\omega}, \omega \in \Omega\right\}$ and a $T_{\hat{D}, \omega} \geq 0$ such that

$$
\phi\left(t, \theta_{-t} \omega, D_{\theta_{-t} \omega}\right) \subset B_{\omega}, \quad \forall t \geq T_{\hat{D}, \omega},
$$

for every tempered random set $\hat{D}=\left\{D_{\omega}, \omega \in \Omega\right\}$ in $X$, then the $R D S(\theta, \phi)$ has a random pullback attractor $\hat{A}=\left\{A_{\omega}, \omega \in \Omega\right\}$.

A set $\hat{\mathcal{B}}=\left\{\hat{B}_{\omega}, \omega \in \Omega\right\}$ in $X$ satisfying the properties required by theorem 3.2 is called a (random) pullback absorbing set of the RDS $(\phi, \theta)$ in $X$.

The pullback attraction in the definition of a random attractor is a form of pathwise convergence. It is known that a random attractor also attracts in the usual forwards sense in the weaker convergence in probability, i.e. given $\epsilon>0$,

$$
\lim _{t \rightarrow+\infty} P\left(\operatorname{dist}_{X}\left(\phi\left(t, \omega, D_{\omega}\right), A_{\theta_{t} \omega}\right)>\epsilon\right)=0 .
$$

\section{Flattening in random dynamical systems}

Definition 4.1. An $\operatorname{RDS}(\phi, \theta)$ on a Banach space $X$ is said to be pullback flattening if for every tempered random bounded set $\mathcal{B}=\left\{B_{\omega}, \omega \in \Omega\right\}$ in $X, \epsilon>0$ and $\omega \in \Omega$ there exists a $T_{0}(\mathcal{B}, \epsilon, \omega)>0$ and a finite-dimensional subspace $X_{\epsilon}$ of $X$ such that

(i) $\bigcup_{t \geq T_{0}} P_{\epsilon} \phi\left(t, \theta_{-t} \omega, B_{\theta_{-} \omega}\right)$ is bounded, and

(ii) $\left\|\left(I-P_{\epsilon}\right)\left(\bigcup_{t \geq T_{0}} \phi\left(t, \theta_{-t} \omega, B_{\theta_{-t}} \omega\right)\right)\right\|_{X}<\epsilon$,

where $P_{\epsilon}: X \rightarrow X_{\epsilon}$ is a bounded projection and (ii) is understood in the sense that $\left\|\left(I-P_{\epsilon}\right) \phi\left(t, \theta_{-t} \omega, x_{0}\right)\right\|_{X}<\epsilon$ for all $x_{0} \in B_{\theta_{-t} \omega}$ and $t \geq T_{0}$.

Definition 4.2. An RDS $(\phi, \theta)$ on a Banach space $X$ is said to be pullback limitset compact if for every tempered random bounded set $\mathcal{B}=\left\{B_{\omega}, \omega \in \Omega\right\}$ in $X$, 
$\epsilon>0$ and $\omega \in \Omega$ there exists a $T_{1}(\mathcal{B}, \epsilon, \omega)>0$ such that

$$
\gamma\left(\bigcup_{t \geq T_{1}} \phi\left(t, \theta_{-t} \omega, B_{\theta_{-t} \omega}\right)\right)<\epsilon,
$$

where $\gamma$ is a measure of non-compactness defined on the subsets of $X$.

Definition 4.3. A $\operatorname{RDS}(\phi, \theta)$ on a Banach space $X$ is said to be pullback asymptotically compact in $X$ if for every tempered random bounded set $\mathcal{B}=\left\{B_{\omega}\right.$, $\omega \in \Omega\}$ in $X$, each $\omega \in \Omega$ and sequences $t_{k} \rightarrow \infty$ and $x_{k} \in B_{\theta_{-t_{k}} \omega}, k=1,2, \ldots$, the set $\left\{\phi\left(t_{k}, \theta_{-t_{k}} \omega, x_{k}\right), k=1,2, \ldots\right\}$ is precompact in $X$.

Remark 4.4. Brzeźniak \& Li (2002, in press) define an analogous concept of a pullback asymptotically compact RDS with respect to deterministic bounded sets B. In particular, their weaker definition than ours is referred to deterministic bounded sets rather than tempered sets. They are then able to prove that omegalimit sets associated to B are non-empty strictly invariant compact random sets which attract B. Our results, with a stronger definition, which is commonly satisfied in applications, lead to the existence of random attractors and so, in particular, they imply this one.

We will prove the following theorems.

Theorem 4.5. Suppose that, $X$ is a uniformly convex Banach space. The following three properties of a random dynamical system on $X$ are equivalent:

(i) pullback flattening,

(ii) pullback limit-set compact, and

(iii) pullback asymptotically compact.

Proof. We will prove that (i) $\Rightarrow$ (ii), then (ii) $\Rightarrow$ (iii) and finally that (iii) $\Rightarrow$ (i).

— the RDS is pullback flattening $\Rightarrow$ the $R D S$ is pullback limit-set compact

Suppose that the RDS is pullback flattening and consider an arbitrary bounded random set $\mathcal{B}=\left\{B_{\omega}, \omega \in \Omega\right\}$ in $X$. Then for each $\omega \in \Omega$

$$
\begin{aligned}
\gamma\left(\bigcup_{t \geq T_{1}} \phi\left(t, \theta_{-t} \omega, B_{\theta_{-t} \omega}\right)\right) \leq & \gamma\left(P\left(\bigcup_{t \geq T_{1}} \phi\left(t, \theta_{-t} \omega, B_{\theta_{-t} \omega}\right)\right)\right) \\
& +\gamma\left((I-P)\left(\bigcup_{t \geq T_{1}} \phi\left(t, \theta_{-t} \omega, B_{\theta_{-t} \omega}\right)\right)\right) \\
\leq 0 & +\gamma\left(B_{X}(0, \epsilon)\right)=2 \epsilon,
\end{aligned}
$$

where $B_{X}(0, \epsilon)$ is the open ball in $X$ with centre 0 and radius $\epsilon$. Hence, the RDS is pullback limit-set compact.

— the RDS is pullback limit-set compact $\Rightarrow$ the RDS is pullback asymptotically compact.

Suppose that the RDS is pullback limit-set compact and let $\mathcal{B}=\left\{B_{\omega}, \omega \in \Omega\right\}$ be a tempered random bounded set in $X$, for each $\omega \in \Omega$ and $\epsilon>0$ there exists 
$T_{1}(\mathcal{B}, \epsilon, \omega)>0$ such that

$$
\gamma\left(\bigcup_{t \geq T_{1}} \phi\left(t, \theta_{-t} \omega, B_{\theta_{-t} \omega}\right)\right)<\epsilon .
$$

Now, if we choose $\epsilon_{n}:=1 / n$ and define $\tau_{n}:=T_{1}(\mathcal{B}, 1 / n, \omega)$ for $n=1,2, \ldots$, with $0<$ $\tau_{1}<\tau_{2}<\ldots$, we get that

$$
\gamma\left(\bigcup_{t \geq \tau_{n}} \phi\left(t, \theta_{-t} \omega, B_{\theta_{-t} \omega} \omega\right)\right)<\frac{1}{n}, \quad n=1,2, \ldots
$$

From the properties of our measure of non-compactness, it follows that

$$
\gamma\left(\overline{\bigcup_{t \geq \tau_{n}} \phi\left(t, \theta_{-t} \omega, B_{\theta_{-t} \omega}\right)}\right)<\frac{1}{n}, \quad n=1,2, \ldots .
$$

Now the bounded sets $A_{n}(\mathcal{B}, \omega)=\overline{\bigcup_{t \geq \tau_{n}} \phi\left(t, \theta_{-t} \omega, B_{\theta_{-} \omega}\right)}$ are nested, i.e. with $A_{n+1}$ $(\mathcal{B}, \omega) \subset A_{n}(\mathcal{B}, \omega)$ for $n=1,2, \ldots$, thus by lemma 2.11 of Wang et al. (in press) their intersection is a non-empty compact subset of $X$, in other words

$$
\varnothing \neq A_{\infty}(\mathcal{B}, \omega)=\bigcap_{n \geq 1} A_{n}(\mathcal{B}, \omega)=\bigcap_{n \geq 1} \overline{\bigcup_{t \geq \tau_{n}} \phi\left(t, \theta_{-t} \omega, B_{\theta_{-t}}\right)} .
$$

Now consider arbitrary sequences $t_{k} \rightarrow \infty$ and $x_{k} \in B_{\theta_{-t_{k}} \omega}, k=1,2, \ldots$, where $\mathcal{B}=\left\{B_{\omega}, \omega \in \Omega\right\}$ is a tempered random bounded set in $X$. Define $F_{j}(\omega):=$ $\left\{\phi\left(t_{k}, \theta_{-t_{k}} \omega, x_{k}\right), k \geq j\right\}$ and (discarding a finite number of $k$ if necessary) define

$$
n_{j}:=\max \left\{n \in \mathbb{N}: \tau_{n} \leq t_{j}\right\},
$$

thus $n_{j} \rightarrow \infty$ as $j \rightarrow \infty$.

$$
\phi\left(t_{k}, \theta_{-t_{k}} \omega, x_{k}\right) \in \phi\left(t_{k}, \theta_{-t_{k}} \omega, B_{\theta_{-t_{k}} \omega}\right) \subset A_{n_{j}}(\mathcal{B}, \omega),
$$

for all $k \geq j$ and $j=1,2, \ldots$. Thus, $F_{j}(\omega) \subset \overline{F_{j}(\omega)} \subset A_{n_{j}}(\mathcal{B}, \omega)$ for all $k \geq j$ and $j=1,2, \ldots$, so

$$
\gamma\left(\overline{F_{j}(\omega)}\right)<\frac{1}{n_{j}} \rightarrow 0 \quad \text { as } \quad j \rightarrow \infty .
$$

But $F_{j+1}(\omega) \subset F_{j}(\omega)$ for $j=1,2, \ldots$, i.e the sets are nested, thus they have their intersection non-empty and compact with

$$
\varnothing \neq \bar{F}(\omega):=\bigcap_{j \geq 1} \overline{F_{j}(\omega)} \subset A_{\infty}(\mathcal{B}, \omega) .
$$

From this we conclude that the set $F_{1}(\omega):=\left\{\phi\left(t_{k}, \theta_{-t_{k}} \omega, x_{k}\right), k \geq 1\right\}$ is precompact, and thus that the RDS is pullback asymptotically compact.

— the RDS is pullback asymptotically compact $\Rightarrow$ the RDS is pullback flattening.

Suppose that RDS is pullback asymptotically compact and let $\mathcal{B}=B_{\omega}, \omega \in \Omega$ be a tempered random bounded set in $X$.

Let $\omega \in \Omega$ be arbitrary but fixed and consider the set

$$
A_{\infty}(\mathcal{B}, \omega)=\bigcap_{n \geq 1} A_{n}(\mathcal{B}, \omega)=\bigcap_{n \geq 1} \overline{\bigcup_{t \geq \tau_{n}} \phi\left(t, \theta_{-t} \omega, B_{\theta_{-t}}\right)} .
$$

It is clear that, a point $a \in A_{\infty}(\mathcal{B}, \omega)$ if, and only if, there are sequences $t_{k} \rightarrow \infty$ and $x_{k} \in B_{\theta_{-t_{k}} \omega}, k=1,2, \ldots$, such that $\phi\left(t, \theta_{-t} \omega, a_{k}\right) \rightarrow a$ as $k \rightarrow \infty$. 
From the assumed pullback asymptotic compactness of the RDS it is clear that $A_{\infty}(\mathcal{B}, \omega)$ is non-empty. Now consider a sequence $a_{k} \in A_{\infty}(\mathcal{B}, \omega), k=1,2, \ldots$ Then for each $k \geq 1$, there exist sequences $t_{k} \geq k$ and $y_{k} \in \phi\left(t, \theta_{-t_{k}} \omega, B_{\theta_{-t_{k}} \omega}\right)$ such that $\left\|y_{k}-a_{k}\right\| \leq 1 / k$. By the pullback asymptotic compactness of the RDS there is a convergent subsequence (which we can relabel as the original one) such that $y_{k} \rightarrow a \in A_{\infty}(\mathcal{B}, \omega)$. Thus, $A_{\infty}(\mathcal{B}, \omega)$ is both non-empty and compact.

Now suppose that $A_{\infty}(\mathcal{B}, \omega)$ does not pullback attract $\mathcal{B}$. Then there is an $\epsilon_{0}>0$ and sequences $t_{k} \rightarrow \infty$ in $\mathbb{R}^{+}$and $y_{k} \in \phi\left(t_{k}, \theta_{-t_{k}} \omega, B_{\theta_{-t_{k}}}\right)$ such that

$$
\operatorname{dist}\left(y_{k}, A_{\infty}(\mathcal{B}, \omega)\right)>\epsilon_{0}, \quad \forall k \geq 1 \text {. }
$$

Thus by pullback asymptotic compactness of the RDS, there is a convergent subsequence (which we can relabel) such that $y_{k} \rightarrow a \in A_{\infty}(\mathcal{B}, \omega)$. But this contradicts $(4.2)$, thus $A_{\infty}(\mathcal{B}, \omega)$ does in fact pullback attract $\mathcal{B}$. In particular, for any $\epsilon>0$ there is a $T_{\epsilon}=T_{\epsilon}(\epsilon, \mathcal{B}, \omega)>0$ such that

$$
\operatorname{dist}_{X}\left(\phi\left(t, \theta_{-t} \omega, B_{\theta_{-t} \omega}\right), A_{\infty}(\mathcal{B}, \omega)\right)<\frac{\epsilon}{4}, \quad \forall t \geq T_{\epsilon},
$$

in other words

$$
\phi\left(t, \theta_{-t} \omega, B_{\theta_{-t} \omega}\right) \subset B\left(A_{\infty}(\mathcal{B}, \omega), \frac{\epsilon}{4}\right) .
$$

Now $A_{\infty}(\mathcal{B}, \omega)$ is a compact subset of $X$, therefore, there exist $N_{\epsilon}$ points $x_{1}$, $x_{2}, \ldots, x_{N_{\epsilon}}$ in $X$ such that

$$
A_{\infty}(\mathcal{B}, \omega) \subset \bigcup_{i=1}^{N_{\epsilon}} B_{X}\left(x_{i}, \frac{\epsilon}{4}\right)
$$

from which it follows that

$$
\phi\left(t, \theta_{-t} \omega, B_{\theta_{-t} \omega}\right) \subset B_{X}\left(A_{\infty}(\mathcal{B}, \omega), \frac{\epsilon}{4}\right) \subset \bigcup_{i=1}^{N_{\epsilon}} B_{X}\left(x_{i}, \frac{\epsilon}{2}\right), \quad t \geq T_{\epsilon},
$$

in other words

$$
\bigcup_{t \geq T_{\epsilon}} \phi\left(t, \theta_{-t} \omega, B_{\theta_{-t}}\right) \subset \bigcup_{i=1}^{N_{\epsilon}} B_{X}\left(x_{i}, \frac{\epsilon}{2}\right) .
$$

Now let $X_{N_{\epsilon}}:=\operatorname{span}\left\{x_{1}, x_{2}, \ldots, x_{N_{\epsilon}}\right\}$. Since $X$ is uniformly convex, there exists a projection $P_{N_{\epsilon}} X \rightarrow X_{N_{\epsilon}}$ such that $\left\|x-P_{N_{\epsilon}} x\right\|=\operatorname{dist}\left(x, X_{N_{\epsilon}}\right)$ for each $x \in X$. Then

$$
\left\|\left(I-P_{N_{\epsilon}}\right)\left(\bigcup_{t \geq T_{\epsilon}} \phi\left(t, \theta_{-t} \omega, B_{\theta_{-t} \omega}\right)\right)\right\| \leq \frac{\epsilon}{2} \leq \epsilon .
$$

$P_{N_{\epsilon}}\left(\bigcup_{t \geq T_{\epsilon}} \phi\left(t, \theta_{-t} \omega, B_{\theta_{-t} \omega}\right)\right)$ is bounded in $X$ since $\bigcup_{t \geq T_{\epsilon}} \phi\left(t, \theta_{-t} \omega, B_{\theta_{-t} \omega}\right)$ is bounded $X$. Thus the RDS is flattening.

Actually, we can easily prove that (iii) $\Rightarrow$ (ii), i.e. if an RDS is pullback asymptotically compact, then it is pullback limit-set compact. Indeed, from (4.3) we get

$$
\gamma\left(\phi\left(t, \theta_{-t} \omega, B_{\theta_{-t} \omega}\right)\right) \leq \gamma\left(A_{\infty}(\mathcal{B}, \omega)\right) \leq \frac{\epsilon}{4}, \quad \forall t \geq T_{\epsilon}
$$

which means that the RDS is pullback limit-set compact.

Note that, in fact, we only use the uniform convexity of the Banach space $X$ in the last implication. Thus, if $X$ is a general Banach space then we have also proved that 
pullback limit-set compact $\Leftrightarrow$ pullback asymptotically compact

and

pullback flattening $\Rightarrow$ pullback asymptotically compact.

Theorem 4.6. Suppose that an $\operatorname{RDS}(\phi, \theta)$ is pullback flattening and has a random bounded absorbing set $\mathcal{B}$. Then it has a unique random attractor $\mathcal{A}$ with $A_{\omega} \subset B_{\omega}$ for every $\omega \in \Omega$.

Proof. Since the RDS has a random pullback absorbing set $\hat{\mathcal{B}}=\left\{\hat{B}_{\omega}: \omega \in \Omega\right\}$, for any bounded random set $\mathcal{B}$, the RDS is pullback absorbed into $\hat{\mathcal{B}}$ in a finite time, so we need only consider the asymptotic behaviour for the RDS starting in $\hat{\mathcal{B}}$.

In addition, the RDS is flattening, thus by theorem 4.5 it is pullback limit-set compact and from the proof of theorem 4.5, there is a non-empty compact subset $A_{\infty}(\hat{\mathcal{B}}, \omega)$ of $X$ for each $\omega \in \Omega$ defined in $(4.1)$. Define $\hat{A}_{\omega}:=A_{\infty}(\hat{\mathcal{B}}, \omega)$ for each $\omega \in \Omega$ and consider the family of non-empty compact subsets of $X$ defined by

$$
\hat{\mathcal{A}}:=\left\{\hat{A}_{\omega}: \omega \in \Omega\right\} .
$$

We need to show that $\hat{\mathcal{A}}$ is

(i) a random set, i.e. $\omega \mapsto \hat{A}_{\omega}$ is measurable,

(ii) is $\phi$-invariant, i.e. $\phi\left(t, \theta \omega, A_{\omega}\right)=A_{\theta_{t} \omega}$, for all, $t \geq 0$, and

(iii) pullback attracting.

These are proved by standard arguments, which have appeared often in the literature. See, for example, Crauel \& Flandoli (1994) or Crauel et al. (1995) for the first property and Wang et al. (in press) for the other two (where the proofs are given in the deterministic context but are also valid here). Concerning the uniqueness of the random attractor, we observe that, in particular, determininstic compact sets are attracted, so that we can apply corollary 5.8 in Crauel (1999).

It also follows that pullback flattening is a necessary condition for the existence of random attractors since if $A_{\omega}$ is a random attractor, then an $\epsilon$-neighbourhood of $A_{\omega}$ is pullback absorbing and we have equation (4.3).

Corollary 4.7. Let $(\phi, \theta)$ be an RDS on a uniformly convex Banach space $X$. If $(\phi, \theta)$ has a random attractor $A_{\omega}$, then the $R D S$ is pullback flattening.

Remark 4.8. Crauel (2001) proved that a random attractor exists if, and only if, there exists a random compact attracting set. Note that the previous corollary does not give the equivalence between the flattening property and the existence of random attractors, since in theorem 4.6 we also need the existence of a random bounded absorbing set.

\section{Pullback flattening and the random squeezing property}

Infinite-dimensional non-autonomous dynamical systems driven, for example, by almost periodic functions are often very uniform in the driving system variables and thus many concepts and results for autonomous dynamical systems carry over to them (Chepyzhov \& Vishik 2002). This holds for non-autonomous 
generalizations of the flattening property (Wang et al. in press). In contrast, random dynamical systems are highly non-uniform and new ideas are needed to take into account this non-uniformity.

Debussche (1997) wrote the following sufficient condition in order to prove finite Hausdorff dimensionality of random attractors: suppose that an $\operatorname{RDS}(\theta, \phi)$ on a Banach space $(X,\|\cdot\|)$ has a random bounded absorbing set $\mathcal{B}=\left\{B_{\omega}, \omega \in \Omega\right\}$ with $B_{\omega} \subset B_{X}(0, r(\omega))$, for all $\omega$ from a $\theta$-invariant set of full measure and for a tempered random variable $r(\omega)$. Let $P: X \rightarrow P X$ be a finite-dimensional orthogonal projector and write $Q=I-P$.

Non-uniformity in the comparison of higher modes of trajectories can be handled in the following way (Debussche 1997): suppose there exists a $\delta \in(0,1 / 2)$ and a random variable $c(\omega)$ with finite expectation $\mathbb{E}(c)<\ln (1 / \delta)$ such that

$$
\left\|Q\left(\phi\left(1, \theta_{\tau} \omega, x\right)-\phi\left(1, \theta_{\tau} \omega, y\right)\right)\right\| \leq \delta \exp \left(\int_{\tau}^{\tau+1} c\left(\theta_{s} \omega\right) \mathrm{d} s\right)\|x-y\|,
$$

for all $x, y \in B_{\theta_{\tau} \omega}$ and each $\tau \in \mathbb{R}$.

This idea led Flandoli \& Langa (1999) to define the following.

Definition 5.1 (Random squeezing property). Either the high modes are bounded by the low modes

$$
\left\|Q\left(\phi\left(1, \theta_{\tau} \omega, x\right)-\phi\left(1, \theta_{\tau} \omega, y\right)\right)\right\| \leq\left\|P\left(\phi\left(1, \theta_{\tau} \omega, x\right)-\phi\left(1, \theta_{\tau} \omega, y\right)\right)\right\|,
$$

or there exists a squeezing on the difference of two trajectories

$$
\left\|\phi\left(1, \theta_{\tau} \omega, x\right)-\phi\left(1, \theta_{\tau} \omega, y\right)\right\| \leq 2 \delta \exp \left(\int_{\tau}^{\tau+1} c\left(\theta_{s} \omega\right) \mathrm{d} s\right)\|x-y\|,
$$

for all $x, y \in B_{\theta_{\tau} \omega}$ and $c(\cdot)$ a random variable $c(\omega)$ with finite expectation satisfying $\mathbb{E}(c)<\ln (1 / \delta)$.

The random squeezing property has been shown to be a sufficient condition for a determining modes result (Flandoli \& Langa 1999; Langa 2003) and the finitedimensionality of random attractors (Debussche 1997; Langa 2003).

It should be no surprise that pullback flattening is a weaker property than the random squeezing property since, given $\epsilon>0$, the pullback flattening property requires a $T_{0}(\mathcal{B}, \epsilon, \omega)>0$ and an abstract finite-dimensional subspace $X_{N}(\epsilon, \omega)$ of $X$, which in general depend on $\epsilon$ (as well as $\omega$ ), whereas in the random squeezing property the finite-dimensional subspace and projector $P$ are fixed in advance and, in particular, do not depend on the value of $\epsilon>0$. Indeed, counter examples can be constructed in which the pullback flattening property holds, but random squeezing is impossible.

Theorem 5.2. Suppose that an $R D S(\theta, \phi)$ in a Banach space $X$ has a positively invariant bounded random absorbing set $\mathcal{B}=\left\{B_{\omega}, \omega \in \Omega\right\}$, with $B_{\omega}=B_{X}(0, r(\omega))$, where $r(\omega)$ is a tempered random variable.

If $(\theta, \phi)$ satisfies the random squeezing property on $\mathcal{B}$, then it is pullback limitset compact and hence the RDS has a random attractor.

In addition, if $X$ is a uniformly convex Banach space, then the $R D S(\theta, \phi)$ is pullback flattening. 
Proof. For notational convenience, we will write $\phi(1, \omega) B_{\omega}$ for $\phi\left(1, \omega, B_{\omega}\right)$, etc.

Following the argument in theorem 3.1 of Debussche $(1997), \phi(1, \omega) B_{\omega}$ can be covered by $k_{0}$ balls of radius $\mathrm{e}^{-1 / 2} r(\omega)$, in other words

$$
\phi(1, \omega) B_{\omega} \subset \bigcup_{i=1}^{k_{0}} B_{X}\left(a_{i}^{1}, \mathrm{e}^{-1 / 2} r(\omega)\right) .
$$

On the other hand, by the co-cycle property,

$$
\phi\left(n, \theta_{-n} \omega\right) B_{\theta_{-n} \omega}=\phi\left(1, \theta_{-1} \omega\right) \circ \phi\left(1, \theta_{-2} \omega\right) \circ \cdots \circ \phi\left(1, \theta_{-n} \omega\right) B_{\theta_{-n} \omega} .
$$

Thus, $\phi\left(1, \theta_{-n} \omega\right) B_{\theta_{-n} \omega}$ can be covered by $k_{0}$ balls of radius $\mathrm{e}^{-1 / 2} r\left(\theta_{-n} \omega\right)$ and, similarly, each of the balls of this covering can be covered by $k_{0}$ balls of radius $\mathrm{e}^{-2 / 2} r\left(\theta_{-n}, \omega\right)$. Iterating this argument, we obtain

$$
\phi\left(n, \theta_{-n} \omega\right) B_{\theta_{-n} \omega} \subset \bigcup_{i=1}^{k_{0}^{n}} B_{X}\left(a_{i}^{n}, \mathrm{e}^{-n / 2} r\left(\theta_{-n} \omega\right)\right) .
$$

Since $r(\omega)$ is tempered, given $\epsilon>0$ there is an $n_{0}$ with $\mathrm{e}^{-n / 2} r\left(\theta_{-n} \omega\right)<\epsilon$ for $n \geq n_{0}$, so that $\phi\left(n, \theta_{-n} \omega\right) B_{\theta_{-n} \omega}$ can be covered by a finite number of balls of radius less than $\epsilon$.

But for $t=n+s, s \geq 0$, by the positive invariance of $B_{\omega}$ and the co-cycle property

$$
\phi\left(t, \theta_{-t} \omega\right) B_{\theta_{-t} \omega}=\phi\left(n, \theta_{-n} \omega\right) \circ \phi\left(s, \theta_{-t} \omega\right) B_{\theta_{-t} \omega} \subset \phi\left(n, \theta_{-n} \omega\right) B_{\theta_{-n} \omega},
$$

for all $t \geq n_{0}$. Thus

$$
\gamma\left(\bigcup_{t \geq n_{0}} \phi\left(t, \theta_{-t} \omega\right) B_{\theta_{-t} \omega}\right)<\epsilon,
$$

so the RDS is thus pullback limit-set compact.

Finally, if $X$ is a uniformly convex Banach space, then theorem 4.5 implies that the RDS is pullback flattening.

\section{(a) A sufficient condition for pullback flattening}

In our abstract theory, the flattening property is equivalent to a RDS to be pullback limit-set compact, so that the last property could be used in order to prove a system to be flattening. However, in many applications, the flattening can be proved directly, and actually flattening and squeezing properties can often be established by related arguments, in the sense that the same projectors can often be used for both the random squeezing property and the pullback flattening, as will be seen in $\S 6$.

Here, we give a general result in this direction, which is also useful for nonautonomous deterministic PDEs and can be used directly for applications involving stochastic PDEs.

Suppose that $(\theta, \phi)$ on a Banach space $X$ is an ergodic RDS with a positively invariant random absorbing set $\mathcal{B}=\left\{B_{\omega}, \omega \in \Omega\right\}$ such that $B_{\omega} \subset B_{X}(0, r(\omega))$, $\mathbb{P}$-a.s., where $r(\omega)$ is a tempered random variable. Let $P: X \rightarrow P X$ be a finitedimensional orthogonal projector with $Q:=I-P$.

Definition 5.3 (Higher mode inequality). There exist a $\delta \in(0,1)$, an orthogonal projector $Q$ and a random variable $c(\omega)$ with finite expectation $\mathbb{E}(c)<\ln (1 / \delta)$ 
such that, $\mathbb{P}$-a.s.

$$
\|Q \phi(t, \omega, x)\| \leq \mathrm{e}^{-t / \delta}\|Q x\|+\delta \exp \left(\int_{0}^{t} c\left(\theta_{s} \omega\right) \mathrm{d} s\right),
$$

for all $x \in B_{\omega}$.

A deterministic version for this property, applied to the two-dimensional Navier-Stokes equations, can be found in Constantin et al. (1985). The main difference between the higher mode inequality (5.3) here and its (squeezing) counterpart in the deterministic case is the presence of the factor $\exp \left(\int_{0}^{t} c\left(\theta_{s} \omega\right) \mathrm{d} s\right)$ for which, in general, there is no uniform bound in time. Hence, the pathwise flattening in the random case is much weaker than that which holds in the deterministic framework.

The higher mode inequality (5.3) is a sufficient condition for the flattening property in random dynamical systems.

Theorem 5.4. Suppose that an ergodic $R D S(\theta, \phi)$ on a Banach space $X$ satisfies the higher mode inequality (5.3). Then the RDS satisfies the pullback flattening property, and hence has a random attractor.

Proof. To prove the pullback flattening, first from (5.3) we obtain the inequality

$$
\|Q \phi(1, \omega, x)\| \leq \mathrm{e}^{-1 / \delta}\|Q x\|+\delta \exp \left(\int_{0}^{1} c\left(\theta_{s} \omega\right) \mathrm{d} s\right)
$$

and note that by the co-cycle property

$$
\phi\left(n, \theta_{-n} \omega, x\right)=\phi\left(1, \theta_{-1} \omega, \cdot\right) \circ \phi\left(1, \theta_{-2} \omega, \cdot\right) \circ \cdots \circ \phi\left(1, \theta_{-n} \omega, x\right),
$$

for all $x \in B_{\theta_{-n} \omega}$.

Consider a point $x_{n} \in B_{\theta_{-n} \omega}$ for a given fixed $n>2$. Then there exists $x_{1} \in B_{\theta_{-1} \omega}$ with $x_{1}=\phi\left(1, \theta_{-2} \omega, \cdot\right) \circ \cdots \circ \phi\left(1, \theta_{-n} \omega, x_{n}\right)$, which satisfy

$$
\left\|Q \phi\left(n, \theta_{-n} \omega, x_{n}\right)\right\|=\left\|Q \phi\left(1, \theta_{-1} \omega, x_{1}\right)\right\| .
$$

Then, using (5.1) iteratively, we obtain

$$
\begin{gathered}
\left\|Q \phi\left(n, \theta_{-n} \omega, x_{n}\right)\right\| \leq \mathrm{e}^{-1 / \delta}\left\|Q x_{1}\right\|+\delta \exp \left(\int_{-1}^{0} c\left(\theta_{s} \omega\right) \mathrm{d} s\right) \\
=\mathrm{e}^{-1 / \delta}\left\|Q \phi\left(1, \theta_{-2} \omega, x_{2}\right)\right\|+\delta \exp \left(\int_{-1}^{0} c\left(\theta_{s} \omega\right) \mathrm{d} s\right)
\end{gathered}
$$

where $x_{2} \in B_{\theta_{-2} \omega}$ with $x_{2}=\phi\left(1, \theta_{-3} \omega, \cdot\right) \circ \cdots \circ \phi\left(1, \theta_{-n} \omega, x_{n}\right)$. Then,

$$
\begin{aligned}
& \mathrm{e}^{-1 / \delta}\left\|Q \phi\left(1, \theta_{-2} \omega, x_{2}\right)\right\|+\delta \exp \left(\int_{-1}^{0} c\left(\theta_{s} \omega\right) \mathrm{d} s\right) \\
& \leq \mathrm{e}^{-1 / \delta}\left(\mathrm{e}^{-1 / \delta} \| Q \phi\left(1, \theta_{-3} \omega, x_{3} \|+\delta \exp \left(\int_{-2}^{-1} c\left(\theta_{s} \omega\right) \mathrm{d} s\right)\right)\right. \\
& \quad+\delta \exp \left(\int_{-1}^{0} c\left(\theta_{s} \omega\right) \mathrm{d} s\right) .
\end{aligned}
$$


Thus, in $n$ steps we obtain

$$
\begin{aligned}
\left\|Q \phi\left(n, \theta_{-n} \omega, x_{n}\right)\right\| & \leq \mathrm{e}^{-n / \delta}\left\|Q x_{n}\right\|+\delta \sum_{j=0}^{n-1}\left(\mathrm{e}^{-n / \delta}\right)^{j} \exp \left(\int_{-j-1}^{-j} c\left(\theta_{s} \omega\right) \mathrm{d} s\right) \\
& \leq \mathrm{e}^{-n / \delta}\left\|Q x_{n}\right\|+\delta \sum_{j=0}^{n-1}\left(\mathrm{e}^{-n / \delta}\right)^{j} \exp \left(\int_{-j-1}^{0} c\left(\theta_{s} \omega\right) \mathrm{d} s\right),
\end{aligned}
$$

By the ergodicity of $\theta_{t}$ and the temperedness of $r(\omega)$ for $n$ large enough so that we can also take $j \geq j_{0}$ large enough, we obtain

$$
\exp \left(\int_{-j-1}^{0} c\left(\theta_{s} \omega\right) \mathrm{d} s\right) \leq \exp \left(\mathbb{E}(c)+\epsilon_{1}\right)(j+1) .
$$

Thus, for the expression in (5.2) we have

$$
\begin{aligned}
\left\|Q \phi\left(n, \theta_{-n} \omega, x_{n}\right)\right\| \leq \mathrm{e}^{-n / \delta}\left\|Q x_{n}\right\| & +\delta\left(c_{1} \sum_{j=j_{0}+1}^{\infty} \mathrm{e}^{j\left(-n / \delta+\mathbb{E}(c)+\epsilon_{1}\right)}\right. \\
& \left.+\sum_{j=0}^{j_{0}}\left(\mathrm{e}^{-n / \delta}\right)^{j} \exp \left(\int_{-j-1}^{0} c\left(\theta_{s} \omega\right) \mathrm{d} s\right)\right) .
\end{aligned}
$$

Observing that the infinite series here is convergent, we have proved for some random constant $c_{2}(\omega)$ that

$$
\left\|Q \phi\left(n, \theta_{-n} \omega, x_{n}\right)\right\| \leq \mathrm{e}^{-n / \delta}\left\|Q\left(x_{n}-y_{n}\right)\right\|+c_{2}(\omega) \delta .
$$

Thus, given $\epsilon>0$, we can choose $\delta$ sufficiently small enough and an $n_{0}$ such that for all $x_{n} \in B_{\theta_{-n} \omega}$ and $n \geq n_{0}$.

$$
\left\|Q \phi\left(n, \theta_{-n} \omega, x_{n}\right)\right\| \leq \epsilon,
$$

Now, if we take $t \geq n_{0}+1$ with $t=n+s$ and $s \in[0,1)$, for $x_{t} \in B_{\theta_{-t} \omega}$ we obtain

$$
\left\|\phi\left(t, \theta_{-t} \omega, x_{t}\right)\right\|=\left\|\phi\left(n, \theta_{-n} \omega, x_{n}\right)\right\|<\epsilon,
$$

with $x_{n}=\phi\left(s, \theta_{-t} \omega, x_{t}\right) \in B_{\theta_{s-t} \omega}$, so that pullback flattening holds.

\section{Two examples}

We consider two well-known examples from the literature and indicate briefly how the flattening property can be verified for them. Since both examples satisfy the random squeezing property then the pullback flattening property also holds. The first example just tries to illustrate an already known model where the theory could be applied.

We think our second example is more interesting, as we will show how to prove the existence of random attractors in space $V$ for stochastic twodimensional Navier-Stokes equations without having to show the existence of an absorbing ball in space $D(A)$ (for a deterministic version of this result see Ma et al. (2002)). Note that since the examples involve additive noise, the noise terms disappear on substraction in setting the squeezing estimates. For the flattening estimates we first have to substract an appropriate Ornstein-Uhlenbeck process to convert the stochastic PDE into a random PDE, but we have to do this in any case to prove the existence of an absorbing ball. 


\section{(a) A stochastic reaction-diffusion equation}

The first known example where a random squeezing property is satisfied appears in Debussche (1997), where the following stochastic reaction-difussion equation is studied: let $D \subset \mathbb{R}^{m}, m \leq 3$, be an open bounded set with regular boundary. We consider

$$
\mathrm{d} u=(\Delta u+f(u)) \mathrm{d} t+\sum_{i=1}^{M} \psi_{i} \mathrm{~d} W_{t}^{i},
$$

with $u(x, t)=0$ for $x \in \partial D$, where the $\psi_{i} \in D(\Delta)$, the $W_{t}^{i}$ are independent two-sided scalar Wiener processes on the probability space $(\Omega, \mathcal{F}, P)$ and $f(u)$ is a polynomial with negative higher order coefficient. From Debussche (1997; section 3.1) we easily conclude the random squeezing property, so that theorem 5.2 holds, and thus the flattening property is satisfied for this example.

\section{(b) Random attractor in $V$ for stochastic two-dimensional Navier-Stokes equations}

We use the notation from Temam (1988), in particular the space $H$ with norm $|\cdot|$ and the space $V$ with norm $\|\cdot\|$, and consider the two-dimensional stochastic (or random) Navier-Stokes equations (RNSE) with scalar additive noise

$$
\frac{\partial u}{\partial t}+u \nabla u-\nu \Delta u+\operatorname{grad} p=f+\psi \mathrm{d} W_{t}, \quad \nabla \cdot u=0,
$$

in a two-dimensional torus $\mathcal{O}$ in $\mathbb{R}^{2}$ with periodic boundary conditions, where $W$ is a two-sided scalar Wiener process and $\psi \in D(A)$ and, for simplicity, we assume $\psi$ is an eigenfunction of the Stokes operator. We write this as

$$
\frac{\mathrm{d} u}{\mathrm{~d} t}=A u+B(u, u)+f+\psi \mathrm{d} W_{t},
$$

and assume that the forcing term $f$ does not depend on time.

To set our problem in the usual abstract framework, we consider the following spaces:

$$
\mathcal{V}=\left\{u \in\left(C_{0}^{\infty}(\mathcal{O})\right)^{2} ; \operatorname{div} u=0\right\} .
$$

$H=$ the closure of $\mathcal{V}$ in $\left(L^{2}(\mathcal{O})\right)^{2}$ with norm $|\cdot|$, and inner product $(\cdot, \cdot)$ where for $u, v \in\left(L^{2}(\mathcal{O})\right)^{2}$,

$$
(u, v)=\sum_{j=1}^{2} \int_{\mathcal{O}} u_{j}(x) v_{j}(x) \mathrm{d} x .
$$

$V=$ the closure of $\mathcal{V}$ in $\left(H_{0}^{1}(\mathcal{O})\right)^{2}$ with norm $\|\cdot\|$, and associated scalar product $((\cdot, \cdot))$, where for $u, v \in\left(H_{0}^{1}(\mathcal{O})\right)^{2}$,

$$
((u, v))=\sum_{i, j=1}^{2} \int_{\mathcal{O}} \frac{\partial u_{j}}{\partial x_{i}} \frac{\partial v_{j}}{\partial x_{i}} \mathrm{~d} x .
$$

It follows that $V \subset H \equiv H^{\prime} \subset V^{\prime}$, where the injections are dense.

Let $\bar{z}(t)$ denote the entire solution (i.e. Ornstein-Uhlenbeck process) of the scalar linear SDE

$$
\mathrm{d} \bar{z}_{t}=-\alpha \bar{z}_{t} \mathrm{~d} t+\mathrm{d} W_{t}
$$


for some $\alpha>0$, in other words

and write $z(t)=\psi \bar{z}_{t}$.

$$
\bar{z}_{t}=\int_{-\infty}^{t} \mathrm{e}^{-\alpha(t-s)} \mathrm{d} W_{s}
$$

Let $v=u-z$. Then, $v$ satisfies the random $\mathrm{PDE}$

$$
\frac{\mathrm{d} v}{\mathrm{~d} t}=A v+B(u, u)+f+\alpha z+A z
$$

Let $Q$ be the projection onto the subspace spanned by eigenfunctions of $A, \phi_{j}$, with $j \geq N$ for some $N$. Write $q=Q v$. Multiplying by $-A q$ and integrating over $\Omega$, we get

$$
\begin{aligned}
\frac{1}{2} \frac{\mathrm{d}}{\mathrm{d} t}\|q\|^{2}+\nu|A q|^{2} & =-(B(u, u), A q)-(f, A q)+\alpha(\psi, A q) \bar{z}-(A \psi, A q) \bar{z} \\
& \leq C|u|^{1 / 2}\|u\||A u|^{1 / 2}|A q|+\frac{2}{\nu}|\bar{z}|^{2}\left(|f|^{2}+|\psi|^{2}+|A \psi|^{2}\right)+\frac{3 \nu}{8}|A q|^{2} \\
& \leq C_{1}|u|\|u\|^{2}|A u|+\frac{2}{\nu}|\bar{z}|^{2}\left(|f|^{2}+|\psi|^{2}+|A \psi|^{2}\right)+\frac{\nu}{4}|A q|^{2}
\end{aligned}
$$

Write $R(t)=C_{1}|u|\|u\|^{2}|A u|+\frac{2}{\nu}|\bar{z}|^{2}\left(|f|^{2}+|\psi|^{2}+|A \psi|^{2}\right)$. Then we have

$$
\frac{\mathrm{d}}{\mathrm{d} t}\|q\|^{2}+\nu \lambda_{N}\|q\|^{2} \leq R(t)
$$

which integrates to give

$$
\|q(t)\|^{2} \leq\|q(s)\|^{2} \mathrm{e}^{-\nu \lambda_{N}(t-s)}+\mathrm{e}^{-\nu \lambda_{N} t} \int_{s}^{t} \mathrm{e}^{\nu \lambda_{N} \tau} R(\tau) \mathrm{d} \tau,
$$

from which it follows that

$$
\begin{aligned}
\|q(t)\|^{2} & \leq\|q(s)\|^{2} \mathrm{e}^{-\nu \lambda_{N}(t-s)}+\mathrm{e}^{-\nu \lambda_{N} t} \int_{s}^{t} e^{\nu \lambda_{N} \tau} R(\tau) \mathrm{d} \tau \\
& \leq\|q(s)\|^{2} \mathrm{e}^{-\nu \lambda_{N}(t-s)}+\mathrm{e}^{-\nu \lambda_{N} t}\left(\int_{s}^{t} \mathrm{e}^{\nu \lambda_{N} \tau} \mathrm{d} \tau\right)^{1 / 2}\left(\int_{s}^{t} e^{\nu \lambda_{N} \tau} R(\tau)^{2} \mathrm{~d} \tau\right)^{1 / 2} \\
& \leq\|q(s)\|^{2} \mathrm{e}^{-\nu \lambda_{N}(t-s)}+\frac{1}{\sqrt{\nu \lambda_{N}}}\left(\mathrm{e}^{-\nu \lambda_{N} t} \int_{s}^{t} e^{\nu \lambda_{N} \tau} R(\tau)^{2} \mathrm{~d} \tau\right)^{1 / 2}
\end{aligned}
$$

The higher modes inequality follows from this last inequality and the fact that the random integral has finite expectation using the results in section 5.2 of Flandoli \& Langa (1999). Thus, the stochastic two-dimensional Navier-Stokes has a random attractor in $V$, rather than just in $H$ as shown elsewhere in the literature.

We sincerely wanted to thank the referees for the very useful and detailed reports, which have led to improve the present paper. Partly supported by the Ministerio de Educación y Ciencia project MTM2005-01412 as well by the Programa de Movilidad del Profesorado universitario español y extranjero, grant SAB2004-0146 of the above Ministerio.

\section{References}

Arnold, L. 1998 Random dynamical systems. Berlin, Germany: Springer.

Brézis, H. 1983 Analyse fonctionnelle: theorie et applications. Paris, France: Masson. 
Brzeźniak, Z. \& Li, Y. 2002 Asymptotic behaviour of solutions to the 2D stochastic Navier-Stokes equations in unbounded domains - new developments. In Proc. First Sino-German Conf. in Stochastic Analysis. 28 August-3 September, Beijing, China, pp. 78-111.

Brzeźniak, Z. \& Li, Y. In press. Asymptotic compactness and absorbing sets for 2D stochastic Navier-Stokes equations on some unbounded domains. Trans. Am. Math. Soc.

Chepyzhov, V. V. \& Vishik, M. I. 2002 Attractors for equations of mathematical physics, vol. 49. Providence, RI: American Mathematical Society.

Chueshov, I. \& Lasiecka, I. 2004 Attractors for second-order evolution equations with a nonlinear damping. J. Dynam. Diff. Equat. 16, 469-512. (doi:10.1007/s10884-004-4289-x)

Constantin, P., Foias, C. \& Temam, R. 1985 Attractors representing turbulent flows. Mem. Amer. Math. Soc., vol. 53. Providence, RI: American Mathematical Society.

Crauel, H. 1999 Global random attractors are uniquely determined by attracting deterministic compact sets. Ann. Mat. Pura Appl. 176, 57-72.

Crauel, H. 2001 Random point attractors versus random set attractors. J. Lond. Math. Soc. 63, 413-427.

Crauel, H. \& Flandoli, F. 1994 Attractors for random dynamical systems. Probab. Theory Relat. Fields 100, 365-393. (doi:10.1007/BF01193705)

Crauel, H., Debussche, A. \& Flandoli, F. 1995 Random attractors. J. Dyn. Diff. Equat. 9, 307-341. (doi:10.1007/BF02219225)

Debussche, A. 1997 On the finite dimensionality of random attractors. Stoch. Anal. Appl. 15, 473-491.

Deimling, K. 1985 Nonlinear functional analysis. Berlin, Germany: Springer.

Eden, A., Foias, C., Nicolaenko, B. \& Temam, R. 1994 Exponential attractors for dissipative evolution equations. Chichester, UK: RAM, Wiley.

Flandoli, F. \& Langa, J. A. 1999 Determining modes for dissipative random dynamical systems. Stoch. Stoch. Rep. 66, 1-25.

Flandoli, F. \& Schmalfuss, B. 1996 Random attractors for the 3D stochastic Navier-Stokes equation with multiplicative white noise. Stoch. Stoch. Rep. 59, 21-45.

Foias, C. \& Prodi, G. 1967 Sur le comportement global des solutions non-stationnaires des equations de Navier-Stokes en dimension 2. Rend. Sem. Mat. Univ. Padova 39, 1-34.

Foias, C. \& Temam, R. 1979 Some analytic and geometric properties of the solutions of the Navier-Stokes equations. J. Math. Pure Appl. 58, 339-368.

Foias, C., Manley, O. \& Temam, R. 1988 Modelling of the interaction of small and large eddies in two dimensional turbulent flows. Math. Mod. Numer. Anal. 22, 93-118.

Hale, J. 1988 Asymptotic behavior of dissipative systems. Mathematical Surveys and Monographs. Providence, RI: AMS.

Khanmamedov, A. Kh. 2006 Global attractors for von Karman equations with nonlinear interior dissipation. J. Math. Anal. Appl. 318, 92-101. (doi:10.1016/j.jmaa.2005.05.031)

Ladyzhenskaya, O. 1991 Attractors for semigroups and evolution equations. Cambridge, UK: Cambridge University Press.

Langa, J. A. 2003 Finite-dimensional limiting dynamics of random dynamical systems. Dyn. Syst. $18,57-68$.

Ma, Q., Wang, S. \& Zhong, C. 2002 Necessary and sufficient conditions for the existence of global attractors for semigroups and applications. Indiana Univ. Math. J. 51, 1541-1559. (doi:10.1512/ iumj.2002.51.2255)

Robinson, J. C. 2001 Infinite-dimensional dynamical systems. Cambridge, UK: Cambridge University Press.

Rosa, R. 1998 The global attractor for the 2D Navier-Stokes flow on some unbounded domains. Nonlin. Anal. TMA 32, 71-85. (doi:10.1016/S0362-546X(97)00453-7)

Schmalfuss, B. 1992 Backward cocycle and attractors of stochastic differential equations. In International seminar on applied mathematics-nonlinear dynamics: attractor approximation and global behaviour (ed. V. Reitmann, T. Redrich \& N. J. Kosch), pp. 185-192. Leipzig, Berlin: Teubner. 
Temam, R. 1988 Infinite-dimensional dynamical systems in mechanics and physics. New York, NY: Springer.

Vishik, M. I. 1992 Asymptotic behaviour of solutions of evolutionary equations. Cambridge, UK: Cambridge University Press.

Wang, Y., Zhong, C. \& Zhou, S. In press. Pullback attractors of nonautonomous dynamical systems. Discrete Conts. Dyn. Systems, Ser. A. 